\title{
GEOMETRIC TAMING OF COMPACTA IN $E^{n}$
}

\author{
DAVID G. WRIGHT
}

\begin{abstract}
We investigate $k$-dimensional compacta in $E^{n}(k \leq n-3)$ that satisfy geometric properties. We prove that such a compactum $X$ in $E^{n}$ is tamely embedded if each point of $X$ can be touched by the tip of a cone from the complement of $X$. Furthermore, we show that a $k$-dimensional compactum $Y$ in $E^{n}(k \leq n-3)$ is tame if $Y$ has vertical order $n-k-2$.
\end{abstract}

1. Introduction. About a decade ago several interesting and beautiful results appeared on taming 2-spheres in Euclidean 3-space $E^{3}$ using geometric conditions. Results by Bothe $\left[\mathbf{B o}_{1}\right]$ and Loveland $\left[\mathbf{L}_{1}\right]$ show that a 2 -sphere in $E^{3}$ must be tamely embedded in $E^{3}$ if it has double tangent balls at each of its points. These results were incorporated into an important work of $\mathrm{J}$. W. Cannon $\left[\mathbf{C}_{\mathbf{1}}, \mathbf{C}_{\mathbf{2}}, \mathbf{C}_{\mathbf{3}}\right]$ where he developed the idea of *-taming sets. A consequence of Cannon's work is that a crumpled cube $C$ in $E^{3}$ is a 3-cell if the boundary of $C$ can be touched at each point by a pencil; i.e., if for each point $p$ of the boundary of $C$ there is a solid right circular cone with vertex at $p$ which lies in the closure of the complement of $C$. Recently, Daverman and Loveland $\left[\mathrm{D}-\mathrm{L}_{1}\right]$ showed that a 2 -sphere in $E^{3}$ with uniform interior tangent balls is flat.

All of the obvious generalizations of the above results to higher dimensions are false with the possible exception of the last which is not known. Daverman and Loveland [D-L $\left.\mathbf{L}_{2}\right]$ and Loveland and Wright $[\mathbf{L}-\mathbf{W}]$ have shown that there are wild spheres in $E^{n}(n>3)$ with double tangent balls at each point. Such examples also show that Cannon's work does not have a straightforward generalization.

Another geometric approach to taming theory in $E^{3}$ is to consider the vertical order of a sphere as was done in [J-L, $\mathbf{L}_{2}, \mathbf{L}_{3}$ and $\left.\mathbf{C}-\mathbf{L}\right]$. These results seem to have no generalization to $E^{n}(n>3)$. Daverman's inflation technique [D] can be used to construct wild spheres in $E^{n}(n>3)$ of vertical order two.

The purpose of this paper is to give taming theorems which are in the spirit of the above 3-dimensional results; however, the object to be tamed will be a compactum of appropriate dimension in $E^{n}$.

The author gratefully acknowledges helpful conversations with John Walsh that aided in the preparation of this paper.

2. Definitions and notation. We use $S^{n}, B^{n}$, and $E^{n}$ to denote the $n$-sphere, the $n$-cell, and Euclidean $n$-space, respectively. We use, $\operatorname{dim} X$ to denote the dimension of a space $X$, and if $X \subset E^{n}$, we let dem $X$ denote the dimension of embedding of $X\left[\mathbf{B r}_{1}, \mathbf{B r}_{2}, \mathbf{S}\right]$. If $X$ is a $k$-dimensional compactum in $E^{n}(k \leq n-3)$, we say $X$ is tame or tamely embedded if $\operatorname{dim} X=\operatorname{dem} X$. For polyhedra and Cantor sets

Received by the editors January 12, 1982. Presented at the 790th meeting of the AMS, November 6, 1970 at Austin Texas, under the title Geometric taming of Cantor sets in $E^{n}$. 1980 Mathematics Subject Classification. Primary 57N35, 57N45; Secondary 57N15, 57N75.

Key words and phrases. Tame embeddings, vertical order, topological embeddings of compacta. 
this corresponds with the usual notions of tameness. For $n \neq 4$ it is known that $\operatorname{dim} X=\operatorname{dem} X$ if and only if $E^{n}-X$ is $1-\mathrm{LC}$ at each point of $X$; i.e., for each $x \in X$ and neighborhood $U$ of $x$ in $E^{n}$, there is a smaller neighborhood $V$ of $x$ in $E^{n}$ such that any map $\alpha: S^{1} \rightarrow V-X$ is null-homotopic in $U-X$. We refer the interested reader to $[\mathbf{E}]$ for a deeper discussion.

Let $\mathbf{v}, \mathbf{w}$ be vectors in $E^{n}$. We let $\mathbf{v} \cdot \mathbf{w}$ denote the usual dot product and $\|\mathbf{v}\|$ denote the usual norm. Choose $p \in E^{n}, \mathbf{v}$ a unit vector in $E^{n}, \theta$ an angle between 0 and $\pi / 2$, and $h$ a positive number. We define the cone with tip $p$, angle $\theta$, altitude $h$, and vector $\mathbf{v}$ to be the set

$$
\left\{x \in E^{n} \mid(x-p) \cdot \mathbf{v} \leq h \text { and } \frac{x-p}{\|x-p\|} \cdot \mathbf{v} \geq \cos \theta\right\} .
$$

Let $p$ be a point in a set $X \subseteq E^{n}$. We say that $X$ can be touched by the tip of the cone at $p$ if there is a cone with tip $p$ whose interior misses $X$.

A set $X$ in $E^{n}$ is said to have vertical order $k$ ( $k$ a nonnegative integer) if each line of the form $\{p\} \times E^{1}, p \in E^{n-1}$, meets $X$ in at most $k$ points.

\section{Taming compacta with cones.}

THEOREM 3.1. Let $X$ be a $k$-dimensional compactum in $E^{n}(k \leq n-3)$ so that each point of $X$ can be touched by the tip of a cone. Then $X$ is tame.

LEMMA 3.2. Theorem 3.1 is true in case each point $p$ of $X$ can be touched by the cone of angle $\theta$, altitude $h$, and vector $\mathbf{v}(p)$ provided there is a unit vector $\mathbf{v}$ and a positive angle $\theta^{\prime}<\theta$ so that $\mathbf{v} \cdot \mathbf{v}(p) \geq \cos \theta^{\prime}$ for all $p \in X$.

PROOF OF LEMMA 3.2. Let $a, b$ be real numbers with $0<b-a<h$. We define $X[a, b]=\{x \in X \mid a \leq x \cdot \mathbf{v} \leq b\}$. Since $X$ can be written as the finite union of such closed sets, we will show that $X$ is tame by showing that $X[a, b]$ is tame. Each line in $E^{n}$ parallel to $\mathbf{v}$ meets $X[a, b]$ in at most one point. Hence, by the Klee trick $[\mathbf{K}]$, we may assume that $X[a, b]$ lies in an $(n-1)$-dimensional hyperplane of $E^{n}$. It is now an easy matter to verify that $E^{n}-X[a, b]$ is 1 -LC at each point of $X[a, b]$. We may therefore conclude that $X[a, b]$ is tame for $n \neq 4$. When $n=4, X[a, b]$ is still tame by the Klee trick $[K]$ if $\operatorname{dim} X[a, b]=0$ and by a theorem of Bryant and Sumners $[\mathbf{B r}-\mathbf{S}]$ if $\operatorname{dim} X[a, b]=1$.

LEMMA 3.3. Theorem 3.1 is true in case each point of $X$ can be touched by a cone of angle $\theta$ and altitude $h$.

ProOF. Let $\theta^{\prime}=\theta / 2$. By compactness there are finitely many unit vectors $\mathbf{v}_{1}, \mathbf{v}_{2}, \ldots, \mathbf{v}_{k}$ so that for each unit vector $\mathbf{u}, \mathbf{u} \cdot \mathbf{v}_{i} \geq \cos \theta^{\prime}$ for some $\mathbf{v}_{i}$.

Let $X_{i}$ be the set of all points in $X$ which can be touched by a cone of angle $\theta$, altitude $h$, and vector $\mathbf{w}$ with $\mathbf{w} \cdot \mathbf{v}_{i} \geq \cos \theta^{\prime}$. The set $X_{i}$ is a closed set which satisfies Lemma 3.2 and is, therefore, tame. Since $X$ is the union of the $X_{i}$, we find that $X$ is tame.

PROOF OF THEOREM 3.1. We let $X_{i}$ be the set of all points in $X$ which can be touched by a cone of angle $1 / i$ and altitude $1 / i$. Each $X_{i}$ is a closed subset of $X$ which is tame by Lemma 3.3. Since $X$ is the union of the $X_{i}$ we conclude that $X$ is tame.

Theorem 3.1 might lead one to believe that a low-dimensional compactum $X$ in $E^{n}$ is tame if each point of $X$ can be touched by a straight line segment in $E^{n}$ 
which meets $X$ precisely at the given point. This is not true as will be seen in the next section.

4. Vertical order of compacta. If $P$ is a compact $k$-dimensional subpolyhedron of $E^{n}(k \leq n-2)$, then by a strong general position theorem [Be-R] we may assume that $P$ has vertical order $(n-1)$. This is often done for a knot in 3-space so that the projection onto the plane has no triple points. We now show that this result can be extended to compacta in a very natural way.

THEOREM 4.1. If $X$ is a $k$-dimensional compactum in $E^{n}(k \leq n-2$ if $n \neq 3$ or $k=0$ if $n=3$ ), then there is an arbitrarily small homeomorphism $h$ of $E^{n}$, fixed outside an arbitrary neighborhood of $X$ so that $h(X)$ has vertical order $n-1$.

ProOF. Since $X$ has embedding dimension less than $(n-1)\left[\mathbf{B r}_{1}, \mathbf{S}, \mathbf{E}\right]$, we can find in any neighborhood $U_{0}$ of $X$ an open $\epsilon_{1}$-mapping cylinder neighborhood whose spine is a polyhedron $P_{1}$ in $E^{n}$ of dimension $n-2$. We may assume that $P_{1}$ has vertical order $n-1$. Let $U_{1} \subset U_{0}$ be a neighborhood of $P_{1}$ chosen so that for each vertical line, $l, l \cap U_{1}$ is contained in the union of $(n-1)$ intervals in $l$ each of length less than 1 . Using the mapping cylinder neighborhood, we construct an $\epsilon_{1}$ homeomorphism $h_{1}: E^{n} \rightarrow E^{n}$, fixed outside the mapping cylinder neighborhood, so that $h_{1}(X) \subseteq U_{1}$.

Inductively, we find an $\epsilon_{i+1}$-mapping cylinder neighborhood of $X_{i}=h_{i} \circ h_{i-1} \circ$ $\ldots \circ h_{2} \circ h_{1}(X)$ that is contained in $U_{i}$ and that has a polyhedron $P_{i+1}$ of vertical order $n-1$ as a spine. Let $U_{i+1} \subseteq U_{i}$ be a neighborhood of $P_{i}$ chosen so that for each vertical line $l, l \cap U_{i+1}$ is contained in the union of $(n-1)$ intervals in $l$ each of length less than $1 /(i+1)$. Once again we use the mapping cylinder neighborhood to construct an $\epsilon_{i+1}$-homeomorphism $h_{i+1}: E^{n} \rightarrow E^{n}$ fixing the complement of the mapping cylinder neighborhood and taking $X_{i}$ into $U_{i+1}$.

By choosing the $\epsilon_{i}$ appropriately the limit $h$ of $\left(h_{i} \circ \cdots \circ h_{2} \circ h_{1}\right)$ is an $\epsilon$ homeomorphism for any preassigned $\epsilon$, which is fixed outside the neighborhood $U_{0}$ of $X$. Since $h(X) \subseteq U_{i}$, we conclude that $h(X)$ has vertical order $n-1$.

We use Theorem 4.1 to construct wild embeddings of continua in $E^{n}$ with small vertical order.

THEOREM 4.2. For $k \leq n-3$, there is a wild $k$-dimensional compactum in $E^{n}$ with vertical order $n-k-1$. For $k>0$ the compactum is connected.

ProOF. For $k=0$ we choose a wildly embedded Cantor set in $E^{n}$. By Theorem 4.1 we may assume that the Cantor set has vertical order $n-1$. For $k>0$, let $A$ be an arc in $E^{n-k}$ that contains a wild Cantor set $C$. We may assume that $A$ has vertical order $n-k-1$ in $E^{n-k}$. Let $p$ be a point in $B^{k}$. Then $B^{k} \times C \cup\{p\} \times A$ is a $k$-dimensional continuum in $E^{n}=E^{k} \times E^{n-k}$ with vertical order $n-k-1$.

The examples of tangled continua of Bothe [Bo] and McMillan and Row [M-R] show that a 1-dimensional continuum in $E^{3}$ need not have embedding dimension 1. For those 1-dimensional compacta which do have embedding dimension 1 , the proof of Theorem 4.1 shows that such compacta can be made to have vertical order 2 in $E^{3}$. It would be surprising if tangled continua could have vertical order 2 . We therefore make the following conjecture.

CONJECTURE. A 1-dimensional compactum in $E^{3}$ with vertical order 2 must have embedding dimension 1 . 
5. Vertical order and taming compacta. Theorem 4.2 shows that a wild, $k$ dimensional compactum in $E^{n}(k \leq n-3)$ may have vertical order as small as $n-k-1$. The following theorem states that any smaller vertical order forces the set to be tame.

THEOREM 5.1. Let $Y$ be a $k$-dimensional compactum in $E^{n}(k \leq n-3)$. If $Y$ has vertical order $n-k-2$, then $Y$ is tame.

Theorem 5.1 is a consequence of the following three theorems. Theorem 5.2 is a well-known fact from dimension theory [H-W, p. 93; H]. Theorem 5.3 seems to be known but has not been explicitly stated in the literature.

THEOREM 5.2. Suppose $f$ is a closed mapping of a space $X$ on a space $Y$ so that $f^{-1}(y)$ has at most $k$ points for each point of $Y$. Then $\operatorname{dim} Y \leq \operatorname{dim} X+k-1$.

THEOREM 5.3. Let $X$ be a $k$-dimensional compactum in $E^{n}(k \leq n-3)$ and $P: E^{n} \rightarrow E^{n-1} \times\{0\}$ be the natural projection map. Suppose $\operatorname{dim} P(X) \leq n-3$ and $\operatorname{dim} X \cap P^{-1}(z) \leq 0$ for each $z \in E^{n-1}$. Then $E^{n}-X$ is 1-LC at each point of $X$.

Proof. Let $f$ be a map of $B^{2}$ into $E^{n}$. It will be sufficient to show that $f$ can be approximated arbitrarily closely by a map $\hat{f}$ so that $\hat{f}\left(B^{2}\right) \cap X=\varnothing$. Since $P(X) \times E^{1}$ is $(n-2)$-dimensional, we may assume, without loss of generality, that $f^{-1}\left(P(X) \times E^{1}\right)$ is a Cantor set $C$ in the interior of $B^{2}$. For each point $p$ in $C$ we find a small open $(n-1)$-cell $U$ in $E^{n-1}$ so that $U \times E^{1}$ contains $f(p)$ and $(U \times\{w\}) \cap X=\varnothing$ for some real number $w$ which differs from the last coordinate of $f(p)$ by a small preassigned number. By compactness we find a finite number of pairwise disjoint disks $D_{1}, D_{2}, \ldots, D_{k}$ in $B^{2}$ whose interiors cover $C$ so that the diameters of $f\left(D_{i}\right)$ are small, $f\left(D_{i}\right) \subset U_{i} \times E^{1}$ for some small $(n-1)$-cell $U_{i}$ in $E^{n-1}$, and $\left(U_{i} \times\left\{w_{i}\right\}\right) \cap X=\varnothing$ for real numbers $w_{i}$ which differ from the last coordinate of any point in $f\left(D_{i}\right)$ by a small amount. Let $\hat{f}$ equal $f$ on the complement of the $D_{i}$. We define $\hat{f}$ on the interior of $D_{i}$ by using a vertical homotopy to the level $E^{n-1} \times\left\{w_{i}\right\}$ and then sending the rest of $D_{i}$ into $U_{i} \times\left\{w_{i}\right\}$. $E^{n}$.

THEOREM 5.4. Let $X$ be a compactum as in Theorem 5.3. Then $X$ is tame in

Proof. As was mentioned in $\S 2$, it is well known that the 1 -LC condition implies tame for $n \neq 4$. For $n=4$ Theorem 5.4 is known for the special case when $X$ is 1-dimensional and lies in a hyperplane [Br-S]. John Walsh and the author have been able to use this special case to show that Theorem 5.4 is true for $n=4$ as well [Wa-Wr]. The proof will not be given here.

PROOF OF THEOREM 5.1. Let $P: E^{n} \rightarrow E^{n-1} \times\{0\}$ be the natural projection. By Theorem $5.2 \operatorname{dim} P(Y) \leq n-3$. By Theorem $5.4 Y$ is tame.

\section{REFERENCES}

[Be-R] H. W. Berkowitz and P. Roy, General position and algebraic independence, Geometric Topology, Proc. Geometric Topology Conf. (Park City, Utah, 1974, L. C. Glaser and T. B. Rushing, eds.), Springer-Verlag, New York, pp. 9-15.

[Bo1] H. G. Bothe, Differenzierbare Flächen sind zahm, Math. Nachr. 43 (1970), 161-180.

[Bo2] _- Ein eindimensionales Kompaktum in $E^{3}$, das sich nicht lagetreu in die Mengersche Universalkurve einbetten lässt, Fund. Math. 54 (1964), 251-258. 
[Bri] J. L. Bryant, On embeddings of compacta in euclidean space, Proc. Amer. Math. Soc. 23 (1969), 46-51.

[Br $\left.\mathbf{B r}_{2}\right]$, On embeddings of 1-dimensional compacta in $E^{5}$, Duke Math. J. 38 (1971), 265-270.

[Br-S] J. L. Bryant and D. L. Sumners, On embeddings of 1-dimensional compacta in a hyperplane in $E^{4}$, Pacific J. Math. 33 (1970), 555-557.

[C $\mathbf{C}_{\mathbf{1}}$ J. W. Cannon, *-taming sets for crumpled cubes, I. Basic properties, Trans. Amer. Math. Soc. 161 (1971), 429-440.

$\left[\mathbf{C}_{2}\right] \_,{ }^{*}$-taming sets for crumpled cubes. II. Horizontal sections in closed sets, Trans. Amer. Math. Soc. 161 (1971), 441-446.

[C $\left.\mathbf{C}_{3}\right] \ldots,{ }^{*}$-taming sets for crumpled cubes. III. Horizontal sections in 2-spheres, Trans. Amer. Math. Soc. 161 (1961), 447-456.

[C-L] J. W. Cannon and L. D. Loveland, A 2-sphere in $E^{3}$ with vertically connected interior is tame, Trans. Amer. Math. Soc. 195 (1974), 345-355.

[D] R. J. Daverman, Embeddings of $(n-1)$-spheres in Euclidean n-space, Bull. Amer. Math. Soc. 84 (1978), 377-405.

[D-L L $_{\text {] }}$ R. J. Daverman and L. D. Loveland, Any 2-sphere in $E^{3}$ with uniform interior tangent balls is flat, Canad. J. Math. 33 (1981), 150-157.

[D-L2] - Wildness and flatness of codimension one spheres having double tangent balls, Rocky Mountain J. Math. 11 (1981), 113-121.

[E] R. D. Edwards, Demension theory. I, Geometric Topology, Proc. Geometric Topology Conf. (Park City, Utah, 1974, L. C. Glaser and T. B. Rushing, eds.), Springer-Verlag, New York, pp. 195-211.

[H] W. Hurewicz, Über dimensionserhöhende stetige Abbildungen, J. für Math. 169 (1933), 71-78.

[H-W] W. Hurewicz and H. Wallman, Dimension theory, Princeton Univ. Press, Princeton, N. J., 1941.

[J-L] R. A. Jensen and L. D. Loveland, Surfaces of vertical order 3 are tame, Bull. Amer. Math. Soc. 76 (1970), 151-154.

[K] V. L. Klee, Some topological properties of convex sets, Trans. Amer. Math. Soc. 78 (1955), 30-45.

[L1] L. D. Loveland, $A$ surface is tame if it has round tangent balls, Trans. Amer. Math. Soc. 152 (1970), 389-397.

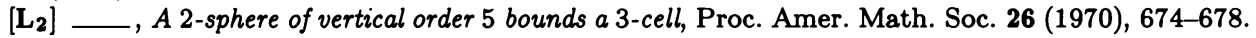

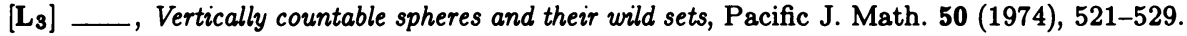

[L-W] L. D. Loveland and D. G. Wright, Codimension one spheres in $\mathbf{R}^{n}$ with double tangent balls, Topology Appl. 13 (1982), 311-320.

[M-R] D. R. McMillan and H. Row, Tangled embeddings of one-dimensional continua, Proc. Amer. Math. Soc. 22 (1969), 378-385.

[S] M. A. Stanko, The embedding of compacta in euclidean space, Mat. Sb. 83 (125) (1970), 234-255; English transl. in Math. USSR Sb. 12 (1970), 234-254. Announcement appeared in Dokl. Akad. Nauk SSSR 86 (1969), 1269-1272 = Soviet Math. Dokl. 10 (1969), 758-761.

[Wa-Wr] J. Walsh and D. G. Wright, Taming compacta in $E^{4}$, Proc. Amer. Math. Soc. 86 (1982), 646-648.

Department of Mathematics, University of Tennessee, KNoXville, TenNESSEE 37996-1300

Current address: Department of Mathematics, Utah State University, Logan, Utah 84322 